Meta

Journal des tradlucteurs

Translators' Journal

\title{
Bibliographie
}

\section{Marcel Marquis}

Volume 17, numéro 4, décembre 1972

URI : https://id.erudit.org/iderudit/003583ar

DOI : https://doi.org/10.7202/003583ar

Aller au sommaire du numéro

Éditeur(s)

Les Presses de l'Université de Montréal

ISSN

0026-0452 (imprimé)

1492-1421 (numérique)

Découvrir la revue

Citer ce document

Marquis, M. (1972). Bibliographie. Meta, 17(4), 248-252.

https://doi.org/10.7202/003583ar

Ce document est protégé par la loi sur le droit d'auteur. L’utilisation des services d'Érudit (y compris la reproduction) est assujettie à sa politique d'utilisation que vous pouvez consulter en ligne.

https://apropos.erudit.org/fr/usagers/politique-dutilisation/
Cet article est diffusé et préservé par Érudit.

Érudit est un consortium interuniversitaire sans but lucratif composé de l'Université de Montréal, l'Université Laval et l'Université du Québec à Montréal. Il a pour mission la promotion et la valorisation de la recherche. https://www.erudit.org/fr/ 


\section{Bibliographie}

\section{BIBLIOGRAPHIES}

Archives de la linguistique française, Paris, A.U.P.E.L.F.-C.N.R.S., 1972, 79 p. Collection de documents relatifs à la langue française, publiés entre 1500 et 1900 , microréédités sous la direction de Bernard Quemada, réalisation technique: Laboratoire de reprographie du Centre de documentation du C.N.R.S.

Bibliographie linguistique 1969, Paris, Klincksieck, 1972, xuvr-528 p.

Complete Catalogue of Books 1972/1973, Toronto, University of Toronto Press, $1972,79 \mathrm{p}$.

Lists all books in print and most of those in preparation through June 1972, and all journals published and distributed by University of Toronto Press. (Communiqué)

VIET, Jean (sous la direction de), Liste mondiale des périodiques spécialisés/ World List of Specialized Periodicals : Linguistique/Linguistics, Paris, Mouton, « Maison des sciences de l'homme», Service d'échange d'information scientifique, Publication série $\mathrm{C}:$ : Catalogues et inventaires », $n^{\circ} 4$, 1972,243 p. [38 F]

\section{DICTIONNAIRES DE LANGUE}

DICTIONNAIRES FRANÇAIS

Grand Larous'se de la langue francaise, 6 vol., Paris, Larousse, 1972, t. II.

GspanN, Lucien, Gallicismes et germanismes... à gogo/Gallizismen und Germanismen... in hülle und fülle, Paris, Didier, 1972, $578 \mathrm{p}$. 
RoBerT, G., Mots et dictionnaires (1798-1878), Paris, Belles Lettres, « Annales littéraires de l'Université de Besançon»(vol. 130), t. VII : Proéminence - rénover, 1972, $400 \mathrm{p}$.

Trésor de la langue française : dictionnaire de la langue $d u 19^{e}$ et du $20^{e}$ siècle, Paris, Klincksieck, 1972, t. I : A-aff, $1016 \mathrm{p}$.

\section{DICTIONNAIRES BILINGUES}

CURREz, M. et F. MorEL, Dictionnaire grec-français du Nouveau Testament (coéd.), Cerf/Delachaux et Niestlé, 1972, 276 p.

GIrard, D., G. Dulona, O. van Oss et C. Guinness, Dictionnaire français-anglais et anglais-français, Paris, Gassell (Garnier), 1464 p., suppl. techn. ill. en coul. de $24 \mathrm{p}$.

Jogo, R. P., Harrap's Concise : français-anglais, anglais-français, Paris, Bordas, «Hippo-Bordas », 1972, 804 p.

Pouliat, P., Dictionnaire français-russe, Paris, Larousse, 1972, 512 p.

ScHNEIDER, L., Dictionnaire esquimau-français, français-esquimau du parler de l'Ungava et contrées limitrophes, Québec, Les Presses de l'Université Laval, 1971, 2 vol., 446 p. et 430 p.

\section{DICTIONNAIRES TECHNIQUES ET SPÉCIALISÉS}

\section{DICTIONNAIRES ANGLAIS}

SAVAGE, George and Harold NewMan, An Illustrated Dictionary of Ceramic Terms, Thames and Hudson, 1972. [\$16.50]

\section{DICTIONNAIRES FRANÇAIS}

Bonnefoux et Paris, le Dictionnaire de la marine à voile, Paris, Éditions de La Courtille (Odéon-Diffusion), 1971, 778 p., avec en supplément, imprimées sur papier «pur chiffon», les 59 planches de l'Encyclopédie de Diderot et d'Alembert concernant la marine et l'évolution des navires. Tirage limité. [2 vol. reliés : 195 F]

Cazelle, H. et A. Feullete, Dictionnaire de la Bible, supplément, Paris, Letouzey et Ané, 1972, fasc. 46 : Prophétisme (suite), 128 p. [48 F]

Cheviluon, M., Petit Dictionnaire du bricoleur, Paris, Bordas, 1972, 128 p.

FLorisoone, M., Dictionnaire des cathédrales de France, Paris, Larousse, 1971, 256 p. $[10,90 \mathrm{~F}]$

Lemionnais, F. et E. Maget, Dictionnaire des échecs, Paris, P.U.F. 1967, 429 p. [\$15.25]

Loтn, B. et Aввє A. Mrchel, Dictionnaire de théologie catholique. Tables générales, Paris, Letouzey et Ané, 1972, fasc. 17 : Stolz à vozanay, 128 p. [45 F] 
PanassiÉ, H. et M. Gautier, Dictionnaire du jazz, préface de L. Armstrong, Paris, Albin Michel, 1972, 368 p. [39 F]

Phíluzon, J. F. Lexique des termes économiques, Paris, Technique et Vulgarisation, «La Documentation pratique », 1972, 177 p., ill. de 5 pl. dépliables:

Pinty, J.-J. et C. GAUTIER, Dictionnaire pratique de mathématiques et statistiques en sciences humaines, Paris, Éditions Universitaires, 1970, 298 p. [\$15.25]

Roman D'Amat (sous la direction de), Dictionnaire de biographie française, Paris, Letouzey et Ané, 1972, fasc. $73:$ Espinas à Eugène, 128 p. et fase. 74 : Eugène à Falconet, 128 p. [48 F chacun]

TRAGaTSCH, E., le Dictionnaire Marabout des voitures de sport et de compétition, 3 vol., Paris, L'Inter, «Marabout Service, série Automobile», $\mathrm{n}^{\text {os }} 154-155$ 156, 1971, 200 p., ill. [chaque vol. broché : 8,90 F]

VersinI, G., Dictionnaire du bridge, Paris, P.U.F., 1968, [\$15.25]

\section{DICTIONNAIRES BILINGUES ET MULTILINGUES}

BAUDET, A., Dictionnaire anglais-français des nouveautés linguistiques, Montréal; Fides, 1971, 198 p. [\$3.95]

Camille, C. et M. Dehaine, Dictionnaire de l'informatique, Paris, Harrap (Bordas), « Harrap », 1971, t. I : Anglais-français, 278 p.; t. II : Françaisanglais, 248 p. [52 F]

Dictionnaire français-anglais et anglais-français des techniques aérospatiales, Paris, Gauthier-Villars, 1971, 410 p. [96 F]

Glossaire européen, Paris, publ. en français, anglais, italien, allemand, $181 \mathrm{p}$. (Dépositaire : Libr. générale de droit et de jurisprudence.)

PIRAUX, H., Dictionnaire anglais-français des termes relatifs à l'électrotechnique, l'électronique et aux applications connexes, $10^{\mathrm{e}}$ éd. mise à jour, Paris, Eyrolles, 1972, $400 \mathrm{p}$.

\section{ENCYCLOPÉDIES, ATLAS ET REPPERTOIRES}

Dictionnaire encyclopédique Lidis, 3 vol., Paris, Lidis, t. I : mai 1972, t. II : décembre 1972, t. III : juin 1973, chaque t. : 368 p.

Duciot, O. et T. Todorov, Dictionnaire encyclopédique des sciences du langage, Paris, Seuil, 1972, 480 p. [45 F]

Encyclopédie de l'art, 6 vol., Paris, Lidis, 1972, t. 5 : Art classique et baroque, $432 \mathrm{p}$.

Encyclopédie du jardinage, Paris, Denoël, «Les Grandes Encyclopédies pratiques $\gg, 1971,512$ p. [43 F]

Encyclopédie thématique Weber, Paris, Weber, 1972, vol. 12 : la Société (3) : sociologie-éducation-sport-jeu, par A. Ahoun, G. Beaune, E. Chanel, G. Meyer, etc., 376 p., 202 ill. [relié : 82 F] 
Grande Encyclopédie Larousse, 20 vol., Paris, Larousse, 400000 sujets, 15000 photos, cartes, dessins, 4 premiers vol. publiés en 1972, $12000 \mathrm{p}$.

Lichine, A., Encyclopédie des vins et des alcools, Paris, Laffont, 1972, 784 p., ill. de 54 cartes.

\section{FORMULAIRES}

Cresson, Bernard, Introduction au français économique, Paris, Didier, « De la langue à la eivilisation française », série Technique, 1972, 144 p. [broché : $10 \mathrm{~F}$, plus 1 série de 10 bandes magnétiques : $660 \mathrm{~F}$ ]

Thimonnier, René, le Code orthographique et grammatical, Paris, Hatier, 1971, 320 p. [35 F]

\section{LINGUISTIQUE}

\section{LINGUISTIQUE FRANÇAISE}

ARCAINI, E., Principes de linguistique appliquée : structures, fonction, transformation, Paris, Payot, « Bibliothèque scientifique », 1972, 304 p. [49,60 F]

BacH, Edmond, Introduction aux grammaires transformationnelles, traduction de Robert Setrich, Paris, Armand Colin, «Linguistique», 1972.

Barbault, M. C. et J.-P. Desclès, Transformations formelles et théories linguistiques, Paris, Dunod, «Documents de linguistique quantitative », $n^{\circ} 11$, $1972,110 \mathrm{p}$.

Charbonneau, René, Étude sur les voyelles nasales du français canadien, Paris, Klincksieck, «Bibliothèque française et romane», série $\mathrm{E}$ : Langue et littérature au Canada, $\mathrm{n}^{\circ} 7,1971, \mathrm{x}-410$ p. [80 F]

Collis, D. R. F., Pour une sémiologie de l'esquimau, Paris, Dunod, « Documents de linguistique quantitative $», \mathrm{n}^{\circ} 14,1971,188$ p. [37 F]

Coyaud, M., Linguistique et documentation, Paris, Larousse, «Langue et Langage $\gg, 1972,176 \mathrm{p}$.

Dubors, Jean et Claude, Introduction à la lexicologie : le dictionnaire, Paris, Larousse, «Langue et Langage», 1971, 208 p.

Genouvrier, E. et Cl. Grumeg, Grammaire nouvelle pour le C.E.I., Paris, Larousse, «Structures de la langue française 》, $\mathrm{n}^{\circ}$ 151, 1972, 96 p.

GladkiJ, A. V. et I. A. Mes'CuK, Éléments de linguistique mathématique, trad. du russe par J. Cohen et D. Hérault, Paris, Dunod, « Monographies de linguistique mathématique $», \mathrm{n}^{\circ} 4,1972,188 \mathrm{p}$.

Malmbera, B., Phonétique générale et romane. Études en allemand, anglais, espagnol et français, Paris, Mouton, «Janua Linguarum », Series Major, $42,1972,478 \mathrm{p}$.

Marmbera, B., les Nouvelles Tendances de la linguistique, $3^{e}$ éd. mise à jour, trad. du suédois par J. Gengoux, Paris, P.U.F., «SUP - Le linguiste», $\mathbf{n}^{\circ} 3,1972,344 \mathbf{p}$. 
Martinet, André, Langue et fonction, Paris, Denoël/Gonthier, "Bibliothèque Médiations », $\mathrm{n}^{\circ} 82,1971,240$ p. [17,50 F]

Mounin, Georges, Clefs pour la sémantique, Paris, Seghers, «Clefs», $\mathrm{n}^{\circ} 16$, 1972,272 p. $[9,90 \mathrm{~F}]$

MounIn, Georges, Intraduction à la sémiologie, Paris, Éditions de Minuit, « Le Sens commun 》, 1971, 244 p. [20 F]

Perrot, Jean, la Linguistique, $9^{e}$ et nouv. éd., Paris, P.U.F., «Que Sais-je?», $\mathrm{n}^{\circ} 570,1972,128$ p. [20 F]

RICHeLle, Marc, l'Acquisition du langage, Paris, Sofedis, « Manuels de psychologie et de sciences humaines $\gg, n^{\circ} 4,1972,215 \mathrm{p}$.

RoBIns, R. H., Linguistique générale, traduction de Simone Delesalle et Paule Guivarch, Paris, Armand Colin, « Linguistique», 1972.

Travaux linguistiques de Prague, 4 (1970). Études de la phonologie, typologie et de la linguistique générale, Paris, Klincksieck, 1972, $236 \mathrm{p}$.

MarCel MarQuis 